\title{
Automated Non-Destructive Imaging and Characterization of the Graphene/hBN Moiré Pattern with Non-Contact Mode AFM
}

\author{
Ardavan Zandiatashbar, ${ }^{*}$ Byong Kim, Young-kook Yoo, and Keibock Lee \\ Park Systems Inc., 3040 Olcott St., Santa Clara, CA 95054 \\ *ardavan@parkafm.com
}

\section{Introduction}

Graphene has attracted researchers' attention because of its unique band gap structure, which allows it to be used in high-mobility semiconductor devices. However, realization of such a graphene-based high-performance device has been challenging due to lack of a suitable substrate. This challenge has been recently addressed by the development of epitaxial growth of graphene on hexagonal boron nitride (hBN) [1, 2]. This material is a suitable substrate for graphene because of its similar hexagonal structure. A moiré pattern is generated as the result of a $\sim 2 \%$ mismatch between graphene and $\mathrm{hBN}$ lattices and the van der Waals interaction between the two layers [3]. The moiré superlattice has periodicity values larger than each of the two materials' lattice constants by two orders of magnitude [4]. The step height of moiré pattern is below one angstrom.

Scanning probe microscopy (SPM) is a key technique for characterization of moiré pattern. In this characterization method, an SPM probe scans the surface of top mono-layer graphene and a three-dimensional surface topography image is generated to detect sub-angstrom surface variations due to the formation of moiré pattern. Optical and electron microscopes can provide images that have high lateral resolution but are limited in providing the required vertical resolution to detect sub-angstrom surface topography. SPM has been known to provide the highest vertical resolution (sub-angstrom) in addition to high lateral resolution [5]. Therefore it is useful for verification of successful fabrication of graphene/hBN devices by epitaxial growth methods. However, SPM has been challenging for two major reasons: there is a steep learning curve for optimization of operating parameters, and the cost is high for the specialized tips required for imaging at the highest resolution. In addition, frictional mode SPM involves mechanical tip-sample engagement, making it a destructive method for characterizing the graphene/hBN devices. Currently most research performed on characterization of the moiré pattern uses destructive SPM modes [1, 2, 4].

Non-contact mode atomic force microscopy (AFM) is a non-destructive SPM technique available since the late 1980s [6]. In order to perform non-contact mode imaging, the tip-sample separation must be controlled accurately. This was one of the limitations at the introduction of this technique. However, thanks to research and development, the technique has reached maturity over the past decade and is now provided as the standard AFM imaging mode by Park Systems.

In this article, the need for easy, non-destructive SPM characterization of moiré patterns has been addressed by an automated non-contact mode AFM imaging technique developed by Park Systems. The commercial name of the product is SmartScan ${ }^{\text {me }}$ Auto Mode. Until today, SPM characterization of the moiré pattern has been challenging, expensive, destructive, and time-consuming. Now with this new solution researchers can easily and reliably perform quality control of fabricated graphene-based devices.

\section{Materials and Methods}

Graphene epitaxy on hBN. For this study, hBN samples were prepared by mechanical cleavage of $\mathrm{BN}$ crystals on a silicon substrate with a $300 \mathrm{~nm} \mathrm{SiO}{ }_{2}$ epilayer. Graphene was grown by CVD epitaxial growth. Detailed information on sample preparation can be found in the reference by Gallagher et al. [7]

Non-contact mode AFM imaging. Non-contact mode imaging is performed by modulating the cantilever's oscillation amplitude at a frequency slightly higher than the resonance frequency of the cantilever in air (far from the sample surface). As the tip approaches the sample surface, tip-sample interaction enters the attractive state, where non-contact mode imaging is performed. By further decreasing tip-sample separation, the interaction switches to the repulsive state (dynamic or tapping mode). The cantilever oscillation phase, which is representing a phase difference between driving signal and output signal, is negative in the attractive state and positive in the repulsive state. Note that this assignment is for AFM systems manufactured by Park Systems. The value and sign of the phase varies depending on the AFM manufacturer.

Variation of amplitude as a function of tip-sample separation is shown in Figure 1 and is referred to as the "A-d curve" hereafter [8]. As shown in Figure 1, when the oscillating cantilever approaches the sample, oscillation amplitude decreases due to tip-sample interaction forces. However, as the tip descends, cantilever amplitude has a small sudden increase in value. This slight jump in amplitude is associated with the changing sign of the phase from negative to positive. This indicates that tip-sample interaction switches from the attractive to the repulsive state. In other words, the cantilever switches from non-contact mode to tapping mode. As the tip continues approaching the surface, the amplitude value approaches zero. If the cantilever is lifted and tip-sample separation increases, the jump from the repulsive to the attractive state occurs again, but at a greater distance from the sample surface. If imaging is performed with cantilever oscillation amplitudes larger (smaller) than the second (first) jump point, imaging is performed in non-contact (tapping) mode, and tip-sample interaction is maintained in the attractive (repulsive) state. If imaging is performed with amplitude values between the first and second jump, the tip-sample interaction will be unstable and switch between attractive and repulsive states, which 
Your Field Emission SEMs for High Contrast, Low Voltage Images From Any Sample

\section{ZEISS GeminiSEM Family}

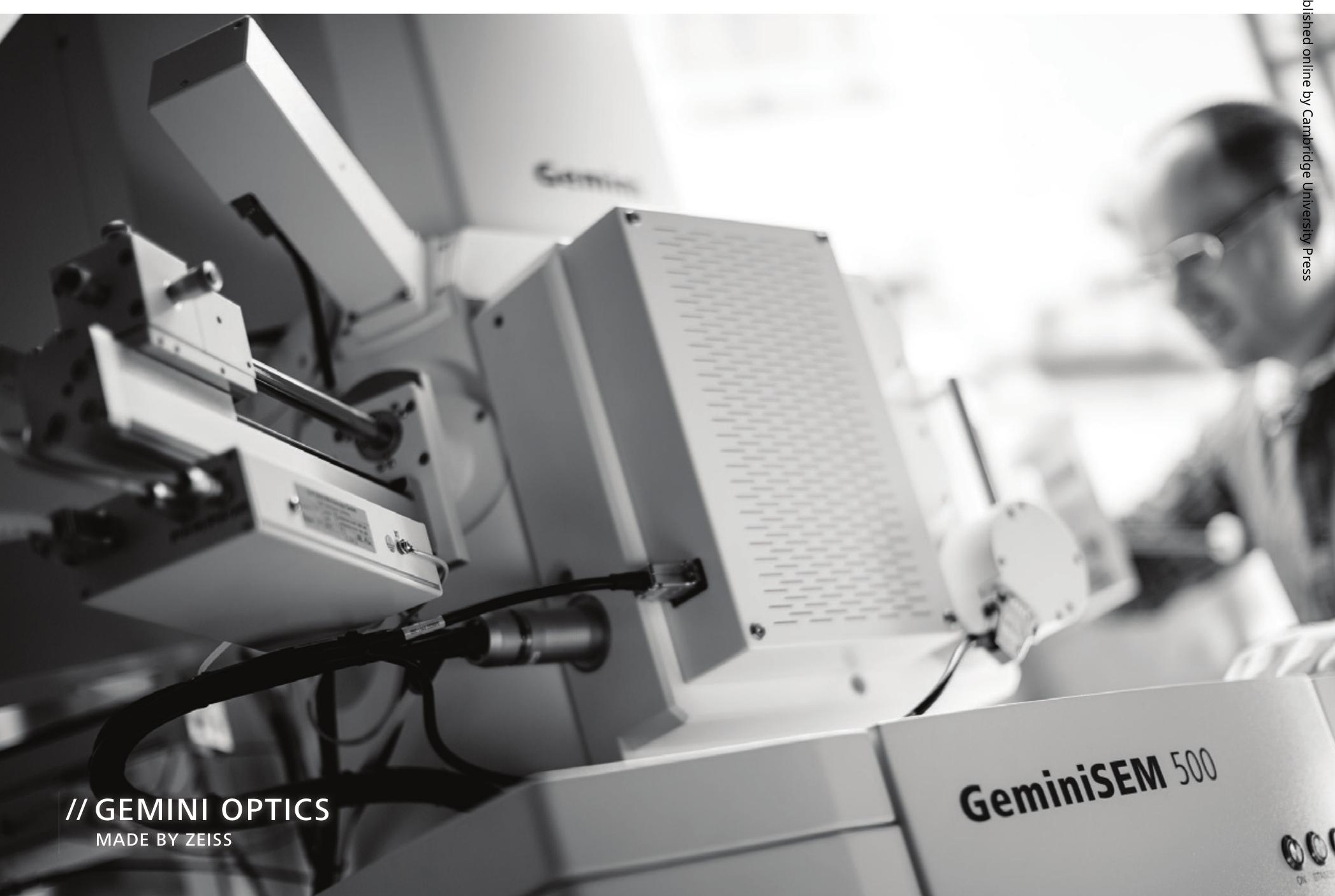

With the ZEISS GeminiSEM family you get a flexible and reliable field emission SEM for your research, industrial lab or imaging facility. You always acquire excellent images from any real world sample. The GeminiSEM family stands for effortless imaging with sub-nanometer resolution and high detection efficiency, even in variable pressure mode. 


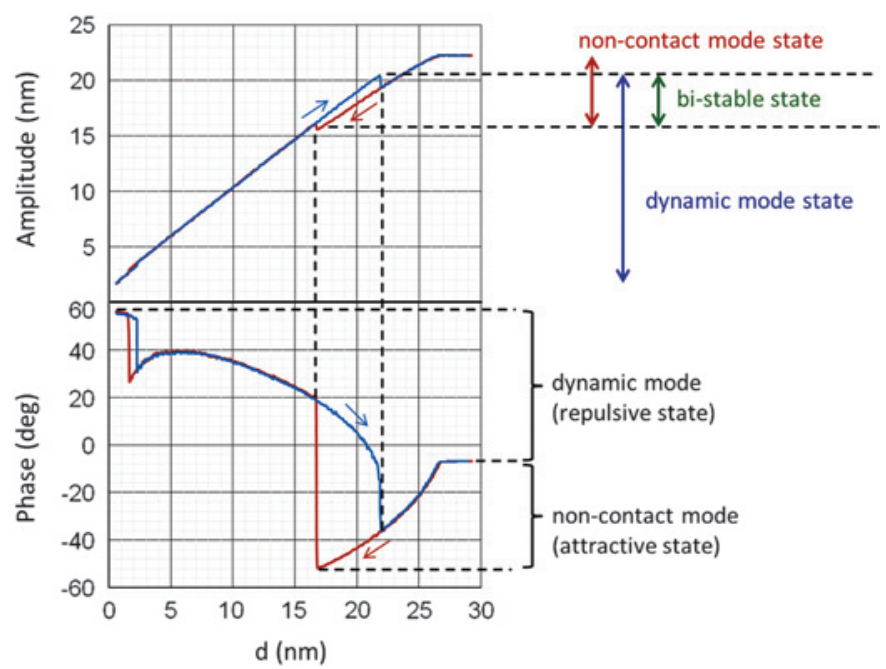

Figure 1: AFM cantilever oscillation amplitude (upper) and phase (lower) vs. tip-sample separation (d) known as the A-d curve for constant z-actuator driving power. The red curves denote the tip approaching the sample and the blue curves show the response when the tip is retracted from the sample surface. [8] (Figure used with permission from Nanoscientific.)

is undesirable for imaging purposes [9]. In these conditions, the feedback system becomes bistable.

SmartScan $^{\text {TM }}$ Auto Mode. SmartScan ${ }^{\text {TM }}$ is the name for new operating software developed by Park Systems for research AFM systems (Figure 2). Auto Mode is one of the major innovations provided by SmartScan ${ }^{\mathrm{Tm}}$ and is used for performing measurements. There are two automation steps after placing the tip and sample, positioning and imaging. In the positioning step, the location of sample surface is found by detecting tip-sample van der Waals interactions. In this step, the tip approaches the sample at a rate of $0.5 \mathrm{~mm} / \mathrm{sec}$ to find the sample surface. Although this is a fairly rapid approach speed, the tip does not come into physical contact with the sample surface. Instead, the tip-sample attractive state and its effect on cantilever oscillation amplitude are used to detect the sample surface. As a result, tip sharpness and the original state of the sample surface are preserved. After detecting the surface, the tip is lifted to a safe distance (about $200 \mu \mathrm{m}$ ) to allow navigating the sample surface and locating the area of interest via a light optical microscope. Because the location of the sample surface is known, the light microscope is brought into focus automatically.

After locating the area of interest, the imaging step begins. During imaging, oscillation frequency, free air and set point amplitudes, feedback control parameters, and scan rate are optimized automatically according to the desired image quality. The user only needs to specify scan size, number of scan lines, and desired image quality. As described above, it is important to maintain accurate tip-sample separation to maintain tip-sample interaction in the attractive state. Accurate tip-sample separation is determined by SmartScan ${ }^{\mathrm{TM}}$ Auto Mode based on the A-d curves shown in Figure 1.

\section{Results}

The graphene/hBN samples were imaged using standard non-contact mode silicon AFM probe with a nominal tip radius of $7 \mathrm{~nm}$ and force constant of $42 \mathrm{~N} / \mathrm{m}$. The tip resonance frequency was set at $316 \mathrm{kHz}$ by the software. The sample surface

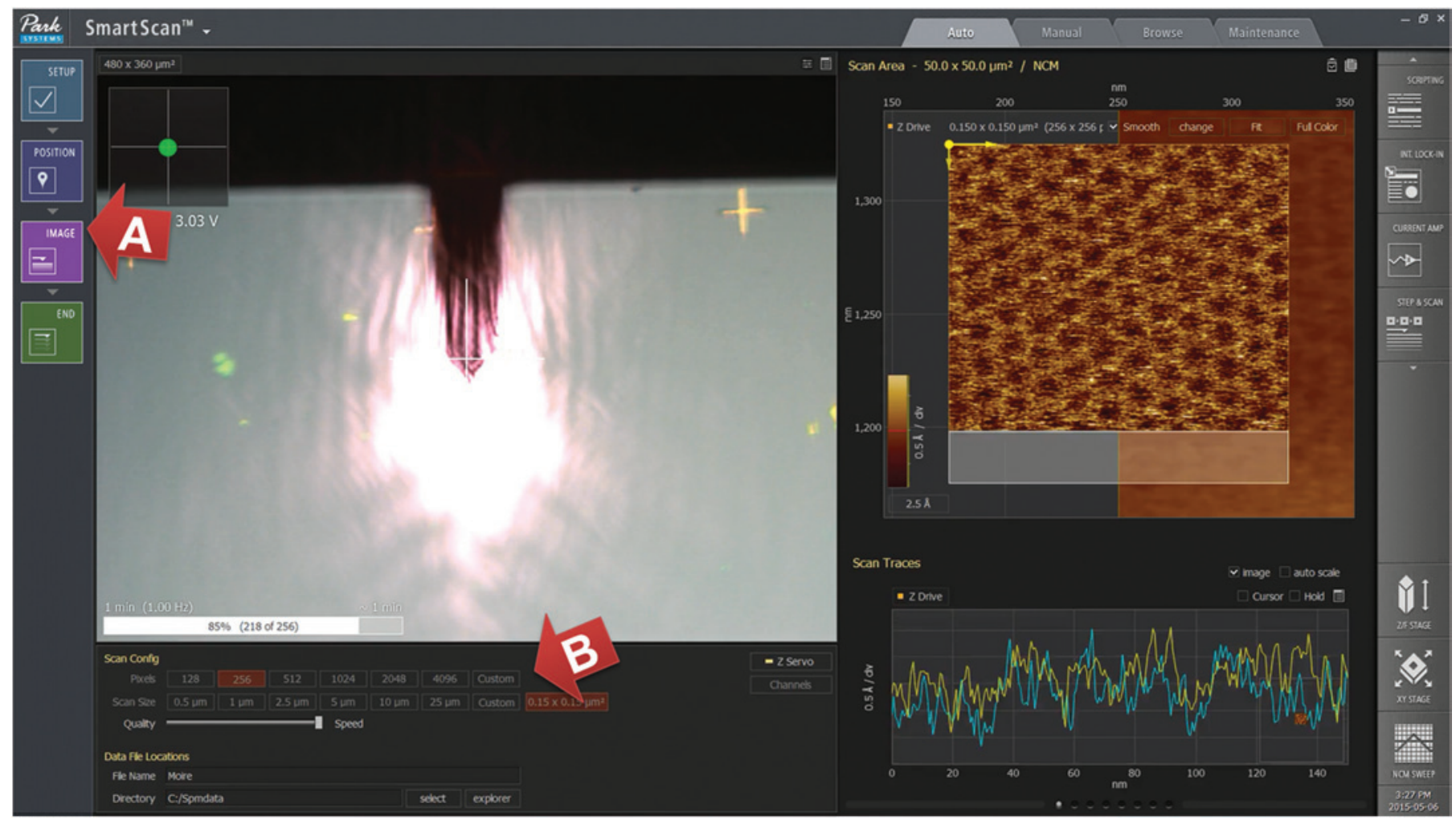

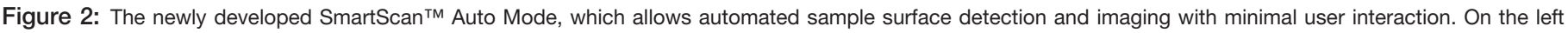

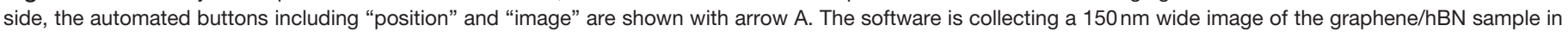
non-contact mode in this screenshot. The user only needs to select scan size, image resolution, and quality/speed from the menu showed by arrow B. 

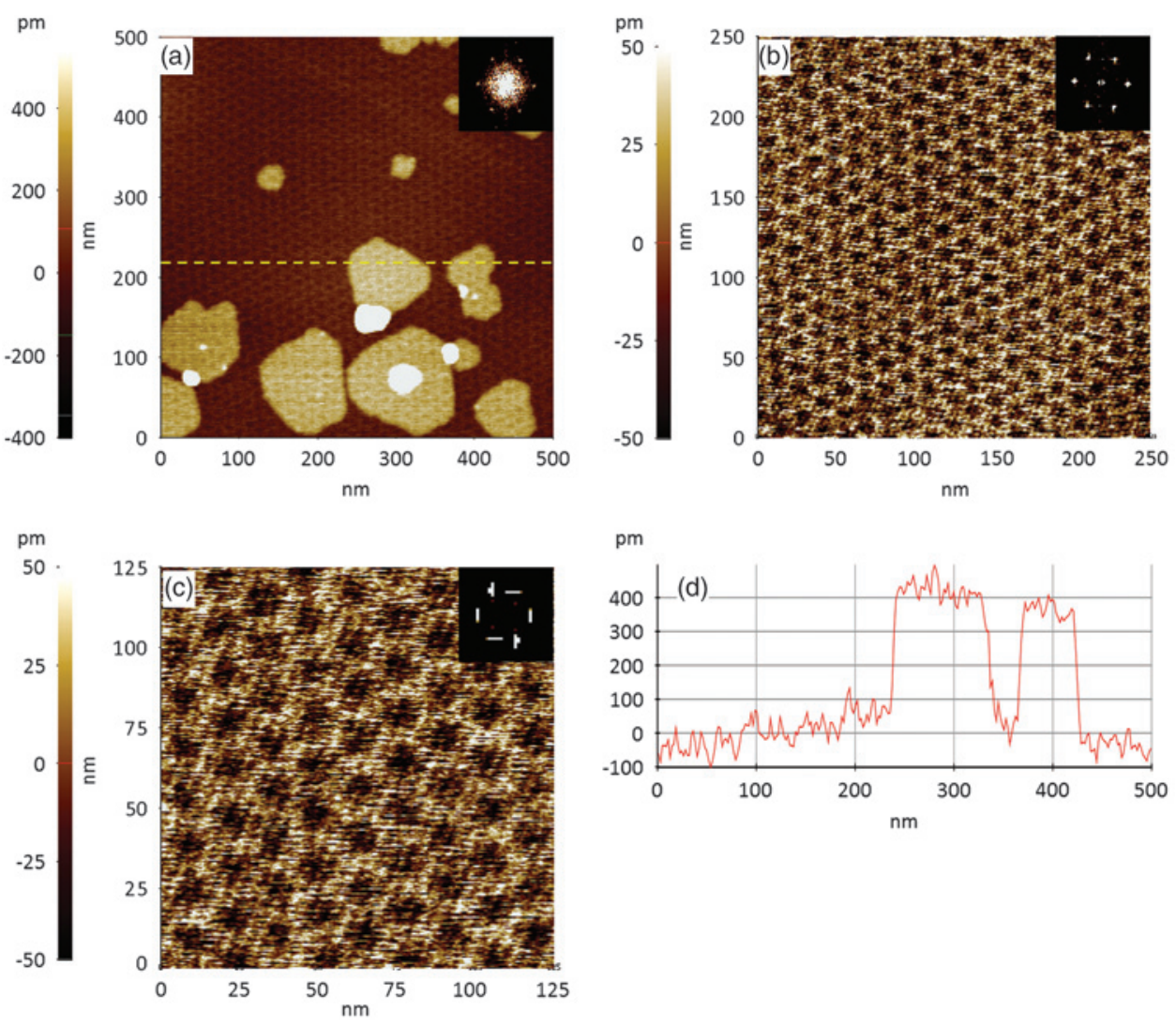

Figure 3: Non-contact mode images of graphene/hBN samples with (a) $500 \mathrm{~nm}$, (b) $250 \mathrm{~nm}$, and (c) $125 \mathrm{~nm}$ scan sizes. No filter has been applied to the images. The insets indicate Fourier transformation of the image taken over the entire image. (d) The profile of the dashed line in the $500 \mathrm{~nm}$ image.

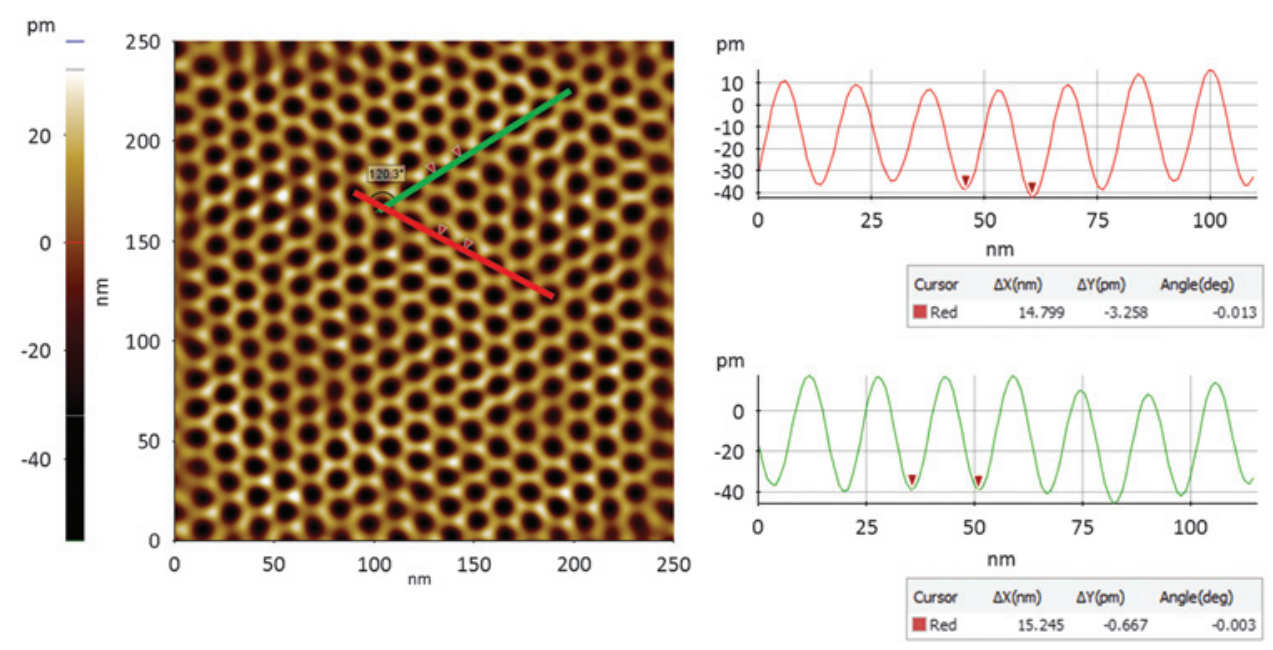

Figure 4: The moiré pattern after an FFT filter was applied to one of the $250 \mathrm{~nm}$ images in Figure $3 \mathrm{~b}$. Line profiles for the red and green lines in the image are shown on the right side. The lattice constant of moiré pattern is measured to be $\sim 15 \mathrm{~nm}$.

was detected by automated software and followed by navigating the sample via light optical microscopy and automated imaging. The images were collected in $500 \mathrm{~nm}, 250 \mathrm{~nm}$, and $125 \mathrm{~nm}$ square sizes. First, lower magnification images were collected, and then image size was decreased for higher magnifications.

Figures $3 a-3 c$ show non-contact mode images of graphene/ hBN samples with $500 \mathrm{~nm}, 250 \mathrm{~nm}$, and $125 \mathrm{~nm}$ scan widths. No filter has been applied to the images. The moiré superlattice of epitaxial graphene is easily distinguishable in all images in the form of a hexagonal pattern. The hexagonal pattern was confirmed by fast Fourier transform (FFT) of AFM images (insets). Grains of a second layer of hBN can be observed in the $500 \mathrm{~nm}$ image as bright islands. To characterize the height of the secondary grains, a line profile of the $500 \mathrm{~nm}$ image was selected as illustrated in Figure $3 \mathrm{~d}$. The height of the secondary grain is $\sim 0.38 \mathrm{~nm}$, which is similar to the thickness of mono-layer graphene. The moiré pattern with similar lattice constant is observable on the secondary grains as well. It is consistent with the height measurement and indicates growth of graphene on the secondary grains.

\section{Discussion}

The moiré superlattice is characterized in Figure 4 by using one of the $250 \mathrm{~nm}$ images. A selective Fourier filter is applied to eliminate additional signal and enable easier characterization of the pattern (Figure 4). For this purpose, only the hexagonal peaks from the FFT of the image are kept, and other signals are eliminated. The lattice constant of the moiré pattern was measured to be $\sim 15 \mathrm{~nm}$. This is consistent with the reported simulation results of $\sim 14 \mathrm{~nm}$ [4], which is two orders of magnitude larger than lattice constants of graphene and hBN. The red and green lines indicate the axes of the superlattice and are used for measuring periodicity in either direction. The peak-to-valley value for each line is below $0.07 \mathrm{~nm}$. The continuity of moiré pattern over the surface indicates successful growth of epitaxial graphene over hBN. The continuity of moiré pattern over the secondary grains also indicates growth of graphene on hBN irrespective of the number of $\mathrm{hBN}$ layers, as seen in the $500 \mathrm{~nm}$ image in Figure $3 a$. It is consistent with the height measurement over the secondary grain. The height measurement of the secondary grains indicates a thickness equivalent to mono-layer graphene. The presence of moiré pattern and thickness of $\sim 0.38 \mathrm{~nm}$ confirms that graphene is present on both primary and secondary grains.

To the knowledge of the authors, imaging the moiré pattern of epitaxial graphene in non-contact mode has not until now been performed. This is of great importance because the 
samples are fragile (low friction between graphene and $\mathrm{hBN}$ ). Therefore, minimized interaction between the tip and sample is necessary to maintain the conditions of the sample during the characterization. There is no mechanical interaction, and the imaging is performed in the ambient atmosphere without the need for a vacuum. Thus, non-contact mode imaging, as a benign characterization technique, can play a key role for characterization of devices fabricated by epitaxial growth of graphene on hBN and other two-dimensional materials.

Preserving tip sharpness is also another advantage of using non-contact mode imaging. In addition to reducing the tip cost, a reliably sharp tip preserves the image quality and improves measurement repeatability. Although finding the correct tip-sample distance for true non-contact mode imaging is challenging and could depend heavily on user experience, using automated software enables these measurements to be performed with minimal user interaction. It also improves repeatability, productivity, and measurement throughput.

A standard non-contact mode probe (PPP-NCHR) was used for this measurement, not the special or super-sharp tip usually required for this sort of high-resolution imaging. In non-contact mode, van der Waals interaction between tip and sample is used to image the moiré superlattice with high resolution and repeatability, even though the moiré pattern lattice constant $(15 \mathrm{~nm})$ is almost twice the nominal tip radius $(7 \mathrm{~nm})$.

\section{Conclusion}

The moiré superlattice of epitaxial graphene grown on $\mathrm{hBN}$ has been imaged in non-contact mode using a recently developed automated AFM. Images were collected using a standard silicon probe with nominal tip radius of $7 \mathrm{~nm}$. Images of the moiré superlattice revealed a lattice constant of $\sim 15 \mathrm{~nm}$, which was verified against simulation values. The newly developed automated AFM improves repeatability, productivity, and throughput. Automated non-contact mode imaging is therefore an efficient characterization technique for quality control of devices fabricated by epitaxial growth, such as graphene/hBN-based devices.

\section{Acknowledgement}

The authors are immensely grateful to David GoldhaberGordon and Patrick Gallagher from Stanford University and Guangyu Zhang from Chinese Academy of Sciences for providing the graphene/hBN sample and fruitful discussions.

\section{References}

[1] W Yang et al., Nature Materials 12 (2013) 792-7.

[2] S Tang et al., Scientific Reports 3(2666) 2013.

[3] B Sachs et al., Phys Rev B 84 (2011) 195414-195422.

[4] CR Woods et al., Nature Physics 10 (2014) 451-6.

[5] GT Smith, Industrial Metrology: Surfaces and Roundness. Springer, New York, 2002.

[6] G Binnig et al., Phys Rev Lett 56(9) 1986) 930-3.

[7] P Gallagher et al., arXiv:1504.05253v1 [cond-mat.mes-hall] (2015) http://arxiv.org/pdf/1504.05253.pdf.

[8] A Zandiatashbar, NanoScientific, Winter (2014) 14-16.

[9] R Garcia and R Perez, Surf Sci Rep 47(6) 2002) 197-301.

\section{The Fastest Way to Pristine: Turbo-Plasma Evactron ${ }^{\circledR}$ Cleaning. Proven by QCM and RGA testing*}

\section{A new generation of Evactron Cleaners clean faster using TMP high vacuum and require little vacuum recovery time.}

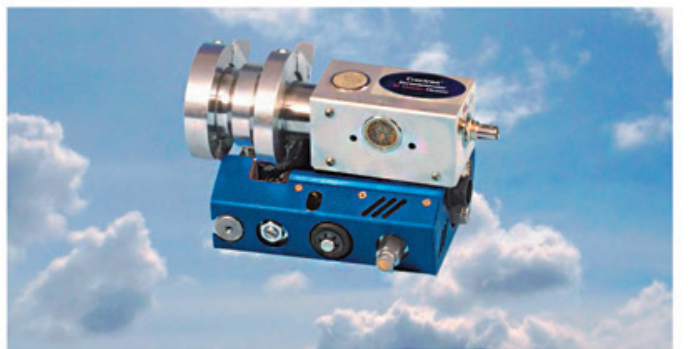

Evactron $^{\circledR}$ Zephyr $^{\text {TM }}$ De-Contaminators Evactron ${ }^{\circledR}$ By XEI Scientific

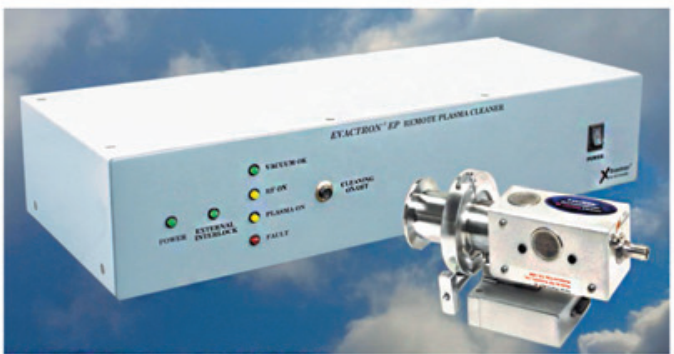

Evactron $^{\circledR}$ EP \& ES Remote Plasma Cleaners

EVACTRON.COM 1-650-369-0133 


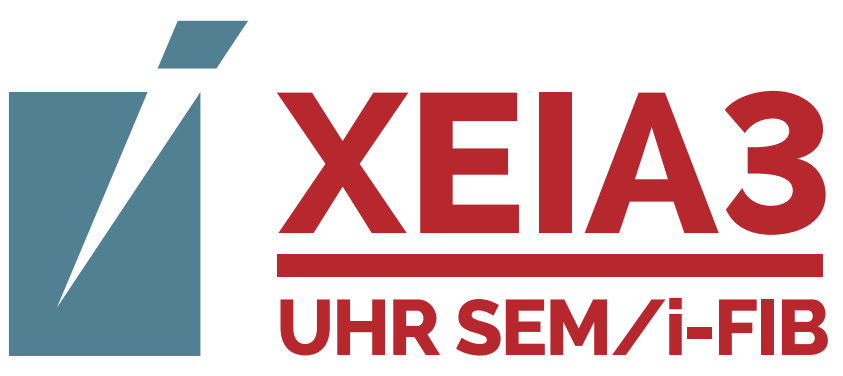

First class

scientific

instrument
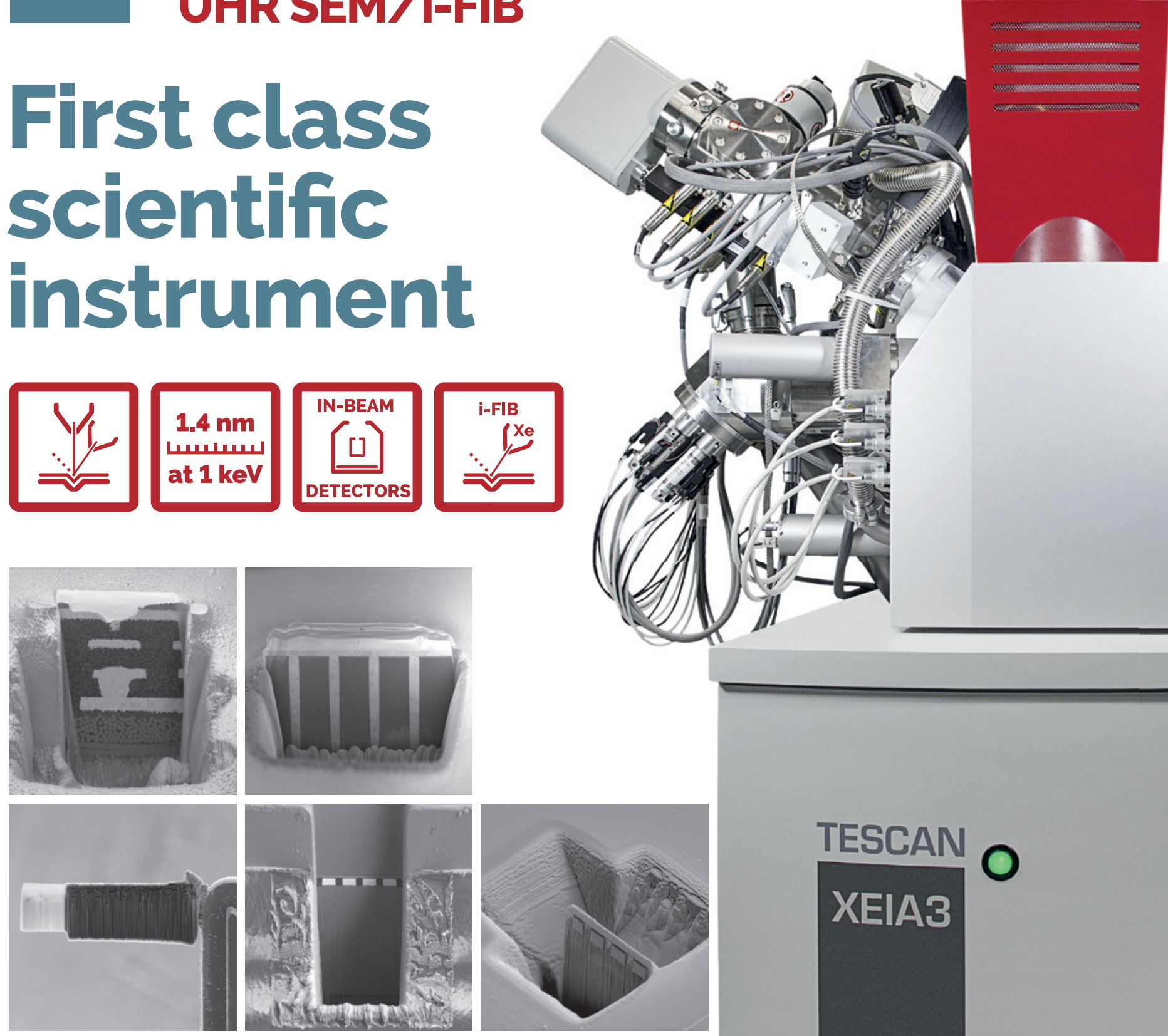

ITTESCAN USA

tescan-usa.com

口涳口

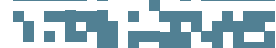

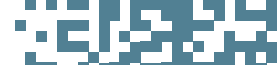

나ㅁㅏㅏ

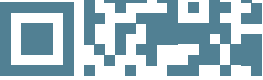

\section{TESCAN XEIA3}

765 Commonwealth D
Warrendale, PA 15086

Tel: (724) 772 - 7433 